\title{
Erratum to: Converging Technologies: A Critical Analysis of Cognitive Enhancement for Public Policy Application
}

\author{
Christos Makridis • Aubrey Wigner
}

Published online: 7 February 2015

(C) Springer Science+Business Media Dordrecht 2015

\section{Erratum to: Sci Eng Ethics (2013) 19:1017-1038 \\ DOI 10.1007/s11948-012-9396-1}

Co-author Aubrey Wigner has been added.

Furthermore, the Acknowledgments should read:

The authors thank Daniel Sarewitz, Michael Crow, and Kyle Whitman for their support, discussions, and inspiration over science policy and technological change.

The online version of the original article can be found under doi:10.1007/s11948-012-9396-1.

C. Makridis $(\bowtie)$

Department of Engineering, Huang Engineering Center, Stanford University, 475 Via Ortega, Stanford, CA 94305-4121, USA

e-mail: cmakridi@stanford.edu

C. Makridis

North American Center for Transborder Study, Wilson Hall, 245 E Orange Mall, Third Floor, PO Box 877103, Tempe, AZ 85287-7103, USA

A. Wigner

Consortium for Science, Policy and Outcomes, Arizona State University, Tempe, AZ 85287, USA

e-mail: aubrey.wigner@asu.edu 